\title{
PLANTAR PRESSURE DISTRIBUTION PATTERNS DURING GAIT IN DIABETIC NEUROPATHY PATIENTS WITH A HISTORY OF FOOT ULCERS
}

\author{
Tatiana Almeida Bacarin, ${ }^{\mathrm{I}}$ Isabel C. N. Sacco, ${ }^{\mathrm{I}}$ Ewald M. Hennig ${ }^{\mathrm{II}}$
}

doi: $10.1590 / \mathrm{S} 1807-59322009000200008$

Bacarin TA, Sacco ICN, Hennig EM. Plantar pressure distribution patterns during gait in diabetic neuropathy patients with a history of foot ulcers. Clinics. 2009:64:113-20.

OBJECTIVE: To investigate and compare the influence of a previous history of foot ulcers on plantar pressure variables during gait of patients with diabetic neuropathy.

INTRODUCTION: Foot ulcers may be an indicator of worsening diabetic neuropathy. However, the behavior of plantar pressure patterns over time and during the progression of neuropathy, especially in patients who have a clinical history of foot ulcers, is still unclear.

METHODS: Subjects were divided into the following groups: control group, 20 subjects; diabetic neuropathy patients without foot ulcers, 17 subjects; and diabetic neuropathy patients with at least one healed foot ulcer within the last year, 10 subjects. Plantar pressure distribution was recorded during barefoot gait using the Pedar-X system.

RESULTS: Neuropathic subjects from both the diabetic neuropathy and DNU groups showed higher plantar pressure than control subjects. At midfoot, the peak pressure was significantly different among all groups: control group $(139.4 \pm 76.4 \mathrm{kPa})$, diabetic neuropathy $(205.3 \pm 118.6 \mathrm{kPa})$ and DNU $(290.7 \pm 151.5 \mathrm{kPa})(\mathrm{p}=0.008)$. The pressure-time integral was significantly higher in the ulcerated neuropathic groups at midfoot (CG: $37.3 \pm 11.4 \mathrm{kPa} . \mathrm{s}$; DN: $43.3 \pm 9.1 \mathrm{kPa} . \mathrm{s} ; \mathrm{DNU}$ : $68.7 \pm 36.5 \mathrm{kPa} . \mathrm{s} ; \mathrm{p}=0.002$ ) and rearfoot (CG: 83.3 \pm 21.2 kPa.s; DN: $94.9 \pm 29.4$ kPa.s; DNU: 102.5 \pm 37.9 kPa.s; $=0.048$ ).

CONCLUSION: A history of foot ulcers in the clinical history of diabetic neuropathy subjects influenced plantar pressure distribution, resulting in an increased load under the midfoot and rearfoot and an increase in the variability of plantar pressure during barefoot gait. The progression of diabetic neuropathy was not found to influence plantar pressure distribution.

KEYWORDS: Biomechanics; Gait; Diabetic Neuropathies; Pressure; Foot ulcer.

\section{INTRODUCTION}

Diabetic peripheral neuropathy (DPN) is one of the most common complications in the progression of diabetes mellitus. It is believed that approximately $50 \%$ of patients with diabetes will develop DPN within 10 to 15 years. ${ }^{1}$ The course of peripheral neuropathy is a progressive degeneration of the peripheral nerves, especially in the

\footnotetext{
I Laboratory of Biomechanics of the Human Movement and Posture; Physical Therapy, Speech and Occupational Therapy Department, Faculdade de Medicina da Universidade de São Paulo/SP, Brazil.

II Laboratory of Biomechanics, University of Duisburg-Essen, Gladbecker Str. Essen, Germany. Email: tbacarin@usp.br

Tel.: 5511 3091-8426 - Fax: $55113091-7462$

Received for publication on July 29, 2008

Accepted for publication October 24, 2008
}

lower limbs, that can cause sensory and motor deficits that affect the biomechanics of the diabetic foot, as seen in ankle kinematics, ${ }^{1-3}$ gait kinetics ${ }^{5,6}$ and plantar pressure distribution. ${ }^{7-10}$

The research literature has reported the existence of a strong association between diabetic neuropathy and higher plantar loads that may be responsible for foot ulceration ${ }^{11-13}$ and re-ulceration. ${ }^{14}$ However, some authors have not found elevated peak pressures for all ulcerated patients. ${ }^{15}$ Therefore, the coexistence of increased peak pressure and the pathogenesis of ulcer formation is considered speculative at best. ${ }^{7}$ Although re-ulceration is common in patients with diabetic neuropathy even with adequate footwear use, ${ }^{16}$ the differences in the distribution patterns of plantar pressure following a completely healed ulcer and its relationship with ulceration recurrence after one year of resolution is 
still unknown.

An ideal approach to study the biomechanical effects of diabetic neuropathy in ulcer development would be a long-term longitudinal study to analyze plantar pressure distribution during gait before and after the ulcer formation. A short-term option is a comparison study of two different groups of neuropathic patients at different stages of the neuropathy to address deficits in sensitivity and previous history of ulceration in order to predict the incidence of reulceration under high plantar pressures.

This study was based on the assumption that the presence of foot ulcers in the clinical history of a diabetic subject is a sign of worsening neuropathy because the development of ulceration is considered the probable result of progressive neurological, vascular and autonomic damage that denotes the advancement of the disease over time. For this reason, the purpose of this study was to investigate and compare the influence of a previous history of foot ulcers on plantar pressure variables during gait. We hypothesized that, even with healed ulcers, diabetic neuropathy patients with a history of ulceration would still show an altered distribution pattern of plantar pressure during gait when compared to patients who had not yet developed ulceration.

\section{MATERIALS AND METHODS}

\section{Subjects}

All subjects were informed about the procedures and signed a written term of free and informed consent that was approved by the ethics committee of the local institution. This cross-sectional study involved three groups: a control group (CG) composed of 20 asymptomatic subjects, a diabetic neuropathy group (DN) of 17 subjects and a diabetic neuropathy group with a history of previous foot ulceration (DNU) composed of 10 subjects.

The diabetic population was recruited through The National Association for Assistance to Diabetics. The control group subjects were employee volunteers from the academic department that conducted this study. Recruiting to the control group was conducted in an attempt to match the anthropometric characteristics of the diabetic neuropathy patients, such as body mass, body mass index, and age (within a decade).

Patients in the diabetic neuropathy groups (DN and DNU) all had a clinical diagnosis of peripheral diabetic neuropathy. The inclusion criteria for both neuropathic groups were diabetes (type 1 or 2) with more than five years since onset and a score higher than six on the Michigan Neuropathy Screening Instrument questionnaire (MNSI-q) for symptoms related to the diabetic neuropathy. ${ }^{17}$
Additionally, subjects were required to have a minimum of two areas where they did not feel a $10 \mathrm{~g}$ monofilament. ${ }^{15}$

For inclusion in the DNU group, the patients were required to present a history of foot ulceration within the last year that was healed at the time of the evaluation.

Subjects were not included if they were over 65 years of age or presented any of these conditions: hallux amputation or partial amputation of the foot; major foot shape alterations by visual inspection; orthopedic disorders of the lower limbs; pain during data acquisition; use of any assistive devices for walking (walking sticks/canes); traumatic ulceration that could be immediately recognized by the subject; Charcot arthropathy confirmed by radiography; or foot ulcers at the time of evaluation.

\section{Experimental Procedures}

First, anthropometric and demographic data were collected and the subjects were asked whether or not they were diabetic, the type and duration of the disease, treatment received for diabetes and the results of their most recent glycemia test. Then, subjects answered the MNSI-q, which is composed of 15 questions regarding typical sensory symptoms of diabetic neuropathy, such as numbness, paresthesia, and pain and burning sensation, and has a maximum score of 13 points. The higher the MNSI score is, the more neuropathic symptoms the patients will report and the worse the neuropathy will be. As part of the screening procedures, a foot inspection was conducted by a single examiner in order to identify foot deformities and to assure similar foot shape in all groups. At this screening stage, tactile sensitivity was also tested as part of the inclusion criteria using $10 \mathrm{~g}$ Semmes-Weinstein monofilaments (Sorri Bauru $^{\circledR}$, Bauru, Brazil ${ }^{18-20}$ on both feet in five areas of each plantar surface: rearfoot, midfoot, lateral forefoot $\left(3^{\text {rd }}\right.$ to $5^{\text {th }}$ metatarsal head), medial forefoot $\left(1^{\text {st }}\right.$ and $2^{\text {nd }}$ metatarsal head) and hallux.

After initial data collection, the subjects walked on a $10 \mathrm{~m}$ walkway at a controlled cadence while wearing only Pedar-X insoles from Novel ${ }^{\circledR}$ System (Munich, Germany) inside anti-skid socks as described in the literature. ${ }^{4}$ The insoles were $2.5 \mathrm{~mm}$ thick and contained a matrix of 99 capacitive pressure sensors with a spatial resolution of 1.6 to $2.2 \mathrm{~cm}^{2}$. Prior to the tests, the insoles were calibrated according to the manufacturer's instructions, and before data acquisition, the zero setting procedure was performed as recommended by Novel. ${ }^{21}$

Before data acquisition, the subjects were instructed to walk freely in the laboratory to reproduce their typical gait and to feel comfortable at the laboratory for a period of approximately five minutes. A digital metronome was 
used to limit gait cadence to a range of 96 to 110 steps per minute in order to avoid large differences in gait cadence among trials and subjects. Gait analysis was performed in the barefoot condition (Pedar-X insoles inside anti-skid socks) to avoid the influence of compounding factors on plantar pressure (e.g., the subject's own shoes). Although the Pedar-X system was designed for evaluating shod gait, it has been used previously by other authors to evaluate barefoot gait ${ }^{22}$. Thus, plantar pressure patterns were studied only by foot-floor interaction. Moreover, the Pedar X system allowed subjects to walk more steps while acquiring the data comparing with the plataform system (EMED, Novel, Munich). While use of the barefoot condition has a high risk of wound formation, the subjects' wearing of shoes would introduce greater variation and variability in plantar pressure data. In view of the increased wound formation risk of the procedure, all subjects were permitted to sit between trials, if needed. A total of 20 steps were recorded for analysis at a sample rate of $50 \mathrm{~Hz}$.

\section{Numerical Analysis}

We divided the plantar surface into the same five areas as described for plantar sensitivity evaluation. The masks used to analyze plantar pressure were adjusted proportionally to the width and length of the foot (Figure 1) and were based on Cavanagh's scheme. ${ }^{23}$ The five areas and their sizes were: M1 rearfoot (27\% of the foot length); M2 midfoot (28\% of the foot length); M3 forefoot (25\% of the foot length), which was divided into two areas: M4 medial forefoot $(55 \%$ of the forefoot width) and M3 lateral forefoot (45\% of the forefoot width); and M5 hallux (the final $20 \%$ of foot length with $33 \%$ of the width).

The variables analyzed for each foot area were peak pressure $(\mathrm{kPa})$ and pressure-time integral (kPa.s), as defined by the impulse of peak pressure over the stance phase. The pressure variables were calculated using the mean value across steps for each trial per subject (approximately 20 steps), and then across trials per subject (approximately 2 trials). Also, the initial and final couple of steps were removed to avoid acceleration and deceleration phases, which could influence our results. The coefficient of variation $(\mathrm{CV})$ was calculated for each plantar pressure variable to represent data variability and was calculated as the standard deviation divided by the mean data values, multiplied by 100 .

\section{Statistical Analysis}

The anthropometric and demographic data were compared among groups using an ANOVA after the data

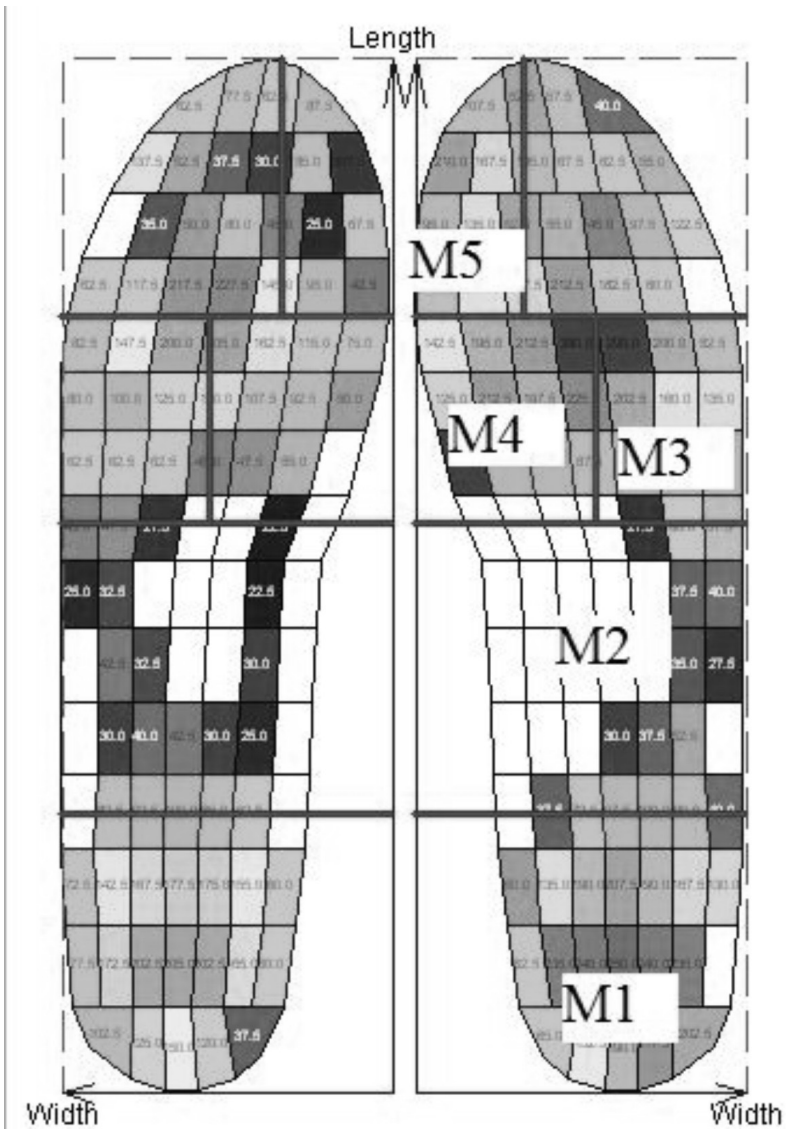

Figure 1 - Masks created in the Creation of Any Mask software (Novel) to analyze plantar pressure in five plantar areas: M1 - Rearfoot; M2 - Midfoot; M3 - Lateral Forefoot; M4 - Medial Forefoot; M5 - Hallux.

distribution had been analyzed by the Shapiro Wilks Test. Categorical variables (e.g., gender, type of diabetes) were compared using Chi-squared test for multiple responses. Clinical variables were compared between diabetic groups using a Student's t-test, and due to their ordinal nature, the MNSI data were compared using the Mann Whitney U-test. Plantar pressure data were compared among groups using ANOVAs when normal distribution was achieved; otherwise, the Kruskal Wallis test was used. The Scheffé tests (for parametric multiple comparisons) and Mann Whitney U-tests (for non-parametric multiple comparisons) were used as post-hoc tests when significant differences were found. An alpha of 5\% was adopted for significant differences.

\section{RESULTS}

Demographic data and the characteristics of the subjects' diabetes are shown in Table 1.

The velocities achieved during gait trials were not different between groups (CG: $64.5 \pm 6.8 \mathrm{~m} / \mathrm{min}$; DN: 64.0 $\pm 7.7 \mathrm{~m} / \mathrm{min}$; DNU: $57.5 \pm 12.3 \mathrm{~m} / \mathrm{min} ; \mathrm{p}=0.2613$ ).

The peak pressure and pressure-time integral are 
shown in Table 2. Peak pressure was significantly different $(\mathrm{p}=0.008)$ among all groups for the midfoot (M2). Neuropathic groups presented statistically higher values than $\mathrm{CG}(\mathrm{p}<0.05)$, and DNU presented the highest values for peak pressure among groups $(\mathrm{p}<0.05)$ (Table 2$)$.

Regarding the plantar-pressure integral, neuropathic subjects presented higher values than control subjects, mainly at the lateral forefoot (M3), midfoot (M2) and rearfoot (M1). Figure 2 represents a summary of the differences observed among groups in the pressure-time integral variable.

DNU subjects presented a higher $\mathrm{CV}$ of pressure data than the CG and DN subjects. Pressure-time integral changes are summarized in Figure 2. The figure of the upper foot represents the DN group in a comparison with the $\mathrm{CG}$ (black arrows). The bottom foot represents the DNU group in a comparison with CG (black arrows) and also in comparison to the DN group (white arrows).

Table 1 - Demographic Data: Control Group (CG), Diabetic Neuropathy (DN) and Ulcerated Diabetic Neuropathy (DNU) Groups

\begin{tabular}{|c|c|c|c|c|}
\hline Variables & $\mathbf{C G}(\mathrm{n}=20)$ & $\mathbf{D N}(\mathrm{n}=17)$ & DNU $(n=10)$ & $\mathbf{p}$ \\
\hline Age $(\text { in years })^{1}$ & $48.7(9.4)$ & $54.7(7.8)$ & $58.2(6.7)$ & 0.1059 \\
\hline $\operatorname{BMI}\left(\mathrm{kg} / \mathrm{m}^{2}\right)^{1}$ & $24.3(2.6)$ & $26.1(4.6)$ & $27(5.5)$ & 0.2445 \\
\hline Male $(\%)^{2}$ & 35 & 47 & 80 & 0.0658 \\
\hline Time since Onset of Diabetes ( in years) ${ }^{3}$ & - & $13.4(8.2)$ & $17.5(9.3)$ & 0.2463 \\
\hline Diabetes Type $2(\%)^{2}$ & _- & 94 & 90 & 0.6125 \\
\hline Mean glycemic index $(\mathrm{mg} / \mathrm{dL})^{3}$ & - & $147.5(58.2)$ & $199.3(60.5)$ & 0.0554 \\
\hline MNSI questionnaire - (median $)^{4}$ & _ & 7 & 7 & 0.8408 \\
\hline
\end{tabular}

The data presented are Mean and Standard Deviation (SD), p-values, Percentage and Median; CG = control group; DN = diabetic neuropathy group; DNU $=$ ulcerated diabetic neuropathy group

${ }^{1}$ ANOVA test, ${ }^{2}$ Chi-square test, ${ }^{3}$ Student t- test, ${ }^{4}$ Mann Whitney test.

Table 2 - Plantar pressure distribution

\begin{tabular}{|c|c|c|c|c|c|}
\hline & Plantar areas & CG & DN & $\mathrm{DNU}$ & $\mathrm{p}$ \\
\hline \multirow{5}{*}{ Peak pressure $(\mathrm{kPa})$} & Hallux $^{2}$ & $\begin{array}{l}306.8(110.7) \\
36 \%\end{array}$ & $\begin{array}{l}305.6(111.7) \\
36 \%\end{array}$ & $\begin{array}{l}269.6(136.7) \\
50 \%\end{array}$ & 0.4650 \\
\hline & Medial forefoot $^{2}$ & $\begin{array}{l}347.5(88.4) \\
25 \%\end{array}$ & $\begin{array}{l}365.4(93.7) \\
25 \%\end{array}$ & $\begin{array}{l}351.6(92.5) \\
26 \%\end{array}$ & 0.6912 \\
\hline & Lateral forefoot ${ }^{1}$ & $\begin{array}{l}328.8(67.5) \\
20 \%\end{array}$ & $\begin{array}{l}367.7(89.2) \\
24 \%\end{array}$ & $\begin{array}{l}367.2(86.2) \\
23 \%\end{array}$ & 0.1096 \\
\hline & Midfoot $^{1}$ & $\begin{array}{l}139.4(76.4) \\
54 \%\end{array}$ & $\begin{array}{l}205.3(118.6) \\
57 \%\end{array}$ & $\begin{array}{l}290.7(151.5) \\
52 \%\end{array}$ & 0.0008 \\
\hline & Rearfoot $^{1}$ & $\begin{array}{l}337.4(95.9) \\
28 \%\end{array}$ & $\begin{array}{l}342.1(76.9) \\
22 \%\end{array}$ & $\begin{array}{l}342.3(119.1) \\
34 \%\end{array}$ & 0.7438 \\
\hline \multirow{5}{*}{ Pressure-time Integral (kPa.s) } & Hallux $^{1}$ & $\begin{array}{l}68.2(24.5) \\
36 \%\end{array}$ & $\begin{array}{l}74.3(26.4) \\
35 \%\end{array}$ & $\begin{array}{l}60.0(24.2) \\
40 \%\end{array}$ & 0.1355 \\
\hline & Medial forefoot ${ }^{2}$ & $\begin{array}{l}97.9(23.2) \\
23 \%\end{array}$ & $\begin{array}{l}110.9(26.5) \\
23 \%\end{array}$ & $\begin{array}{l}110.0(31.7) \\
28 \%\end{array}$ & 0.0403 \\
\hline & Lateral Forefoot $^{2}$ & $\begin{array}{l}97.7(18.4) \\
18 \%\end{array}$ & $\begin{array}{l}119.3(31.8) \\
26 \%\end{array}$ & $\begin{array}{l}125.9(33.4) \\
26 \%\end{array}$ & 0.0003 \\
\hline & Midfoot $^{1}$ & $\begin{array}{l}37.3(11.4) \\
30 \%\end{array}$ & $\begin{array}{l}43.3(9.1) \\
21 \%\end{array}$ & $\begin{array}{l}68.7(36.5) \\
53 \%\end{array}$ & 0.0002 \\
\hline & Rearfoot $^{1}$ & $\begin{array}{l}83.3(21.2) \\
25 \%\end{array}$ & $\begin{array}{l}94.9(29.4) \\
30 \%\end{array}$ & $\begin{array}{l}102.5(37.9) \\
37 \%\end{array}$ & 0.0486 \\
\hline
\end{tabular}

Mean and Standard Deviations (SD) and Coefficient of Variation (\%) and p-values. Control Group (CG); Diabetic Neuropathy (DN) and Ulcerated Diabetic Neuropathy (DNU) Groups. Bold data represent the groups that were different from each other. ${ }^{1}$ Kruskal Wallis test, ${ }^{2}$ ANOVA test. 


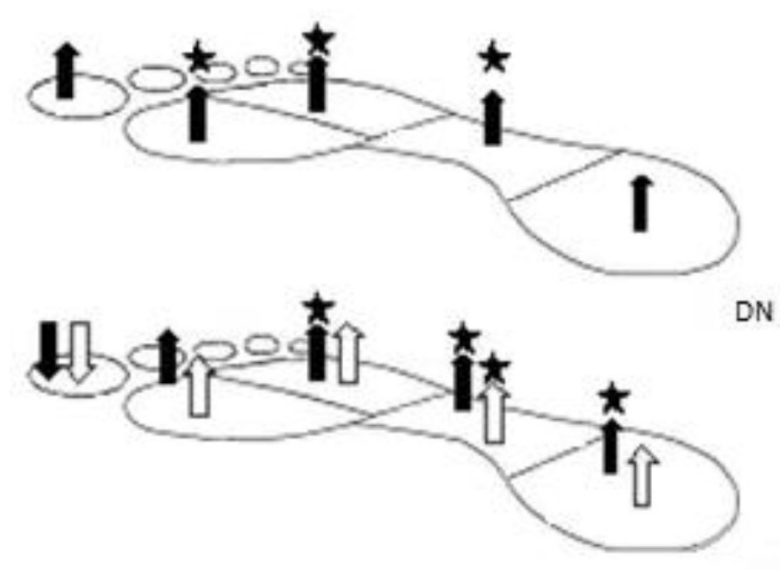

DNU

Figure 2 - Summary of differences in pressure-time integral according to its tendency. Upper panel presentation: comparison between DN and CG (black arrows), showing higher values for DN. Lower panel: comparison between DNU and CG (black arrows) and to DN (white arrows). Black stars represent significant differences $(\mathrm{p}<0.05)$ between groups.

\section{DISCUSSION}

This study investigated plantar pressure distribution during gait, which is influenced by many factors ${ }^{24,25}$ in subjects at different stages of diabetic neuropathy, with $(n=17)$ or without $(n=10)$ a history of foot ulcers, as compared a non-diabetic control group $(n=20)$.

In the present paper, the presence of ulceration in the neuropathic clinical history was assumed to be an indicator of a worsening progression of neuropathy. However, the neuropathic groups were composed of diabetics with similar disease characteristics ( $p>0.05$ ) according to the duration of disease, type of diabetes and diabetic neuropathy symptoms (MNSI). Although the length of disease since onset was not significantly different, the ulcerated group had a higher mean. It has long been known that diabetic neuropathy is a chronic consequence of diabetes mellitus and that the risk of ulceration increases with time. ${ }^{1}$

It was expected that diabetics with advanced complications due to neuropathy, such as a history of foot ulceration, would present more severe symptoms, but this was not confirmed by MNSI scores. Therefore, the assumption that the presence of ulcers is an indicator of a worsening progression of neuropathy is not relevant. In addition, it is already known that good foot care, including daily foot inspection, moisturizing, and appropriate footwear, may prevent ulceration even in the presence of severe neuropathy. Other factors such as sensorial, autonomic and motor dysfunctions may be related to the pathogenesis of ulceration.

The ulcerated diabetic patients may have already developed autonomic and vascular deficits that were not evaluated by the MNSI in this study, but that could have contributed greatly to ulcer formation. The authors recently discussed the lack of significant differences with regard to symptoms between diabetic neuropathic populations with and without a clinical history of foot ulceration. ${ }^{26}$ Other findings, such as nerve conduction velocity and sensory tests, were able to differentiate both populations in the same study. Moreover, development of foot ulceration has been strongly associated with the loss of protective sensations in the diabetic foot. . $19,26,27^{-1}$

The neuropathy groups presented significantly higher values of peak pressures under the midfoot and pressuretime integrals under all plantar areas except under the hallux (Table 2). Some studies have shown changes in plantar pressure distribution patterns following induction of plantar insensitivity. ${ }^{28-30}$ In this study, the loss of protective sensation was not transitory as found by others authors, ${ }^{28,30}$ but was present in the neuropathic subjects and may explain the higher plantar loads found when compared to nonneuropathic subjects.

Comparing both neuropathic groups, the ulcerated subjects showed higher peak pressures under the midfoot and pressure-time integrals under the midfoot and rearfoot. The Charcot condition was excluded in the neuropathic subjects, and this absence was confirmed by radiographic examination; thus, the higher peak pressure at the midfoot in ulcerated diabetics may not be associated with either the Charcot condition or the dropped longitudinal arch among neuropathic subjects. All subjects from the three groups presented an arch index classified as normal. ${ }^{31}$ The higher peak pressure under the midfoot can be explained by the shift of the loading pattern from the lateral toward the medial part of the foot and from the rearfoot to the anterior part of the foot at the roll over process, which is even more evident in ulcerated patients. ${ }^{32}$ Additionally, as noted previously, compensatory musculoskeletal mechanisms developed in neuropathic patients to compensate for their sensory deficit, altering the foot roll over mechanism. ${ }^{33}$

It is important to point out other factors that interfere with plantar pressure distribution, such as foot deformities ${ }^{34}$ and mobility of the foot and ankle joint complex. ${ }^{35-37}$ Diabetic neuropathy causes a progressive alteration in muscle trophism, especially in the intrinsic foot/ankle muscles, an increase in joint rigidity, and an alteration of the collagen structure in the fasciae and muscle tendons due to collagen crosslinking and non-enzymatic glycosylation of keratin. This means that muscles, cartilage, tendons and ligaments will have their structures changed, which will culminate in a limitation of foot mobility. ${ }^{35,36,38}$ This study did not assess the compounding variable of joint mobility. However, we 
did exclude subjects with apparent foot deformity or Charcot arthropathy confirmed by radiography. Furthermore, the literature is not yet clear regarding the relationship between the smaller passive and/or dynamic ankle range of motion and higher plantar pressures, since a recent study showed that despite a significant reduction in the passive range of motion at the ankle joint complex in diabetic subjects, the gait range of motion was indistinguishable from that of control subjects and was not correlated with plantar pressure variables. ${ }^{39}$

Alterations of plantar pressure distribution at the midfoot were also detected in a recent study ${ }^{40}$ showing a possible presence of polymorphism that could not be detected by static radiographies. The subjects evaluated in the present study did not have Charcot arthropathy, but the lateral column of the midfoot could be responsible for raising the loads over their midfoot. ${ }^{40}$

Although there are other factors that can affect plantar pressure distribution, the present study focused on one particular aspect of this chain on plantar pressure alteration: neuropathy progression with the presence of at least one ulcer in the patient's clinical history. It was observed that this factor interferes in the pressure pattern distribution. DNU subjects presented a worse load distribution pattern during gait, overloading the midfoot and the rearfoot. These changes could be associated with foot ulcer pathogenesis or could be an additional alteration after ulcer healing that worsens the biomechanical condition of these patients and their clinical consequences.

The values of plantar pressures found for all areas for each experimental group were not considered to be a risk for foot ulceration based on the thresholds described in the literature: $600 \mathrm{kPa},{ }^{15,41} 1000 \mathrm{kPa}^{42}$ and $1230 \mathrm{kPa} \cdot{ }^{14}$ However, diabetic neuropathic patients can develop ulceration even under normal plantar pressure values. ${ }^{1}$ This factor becomes more important when the biomechanical condition of ulcerated patients is considered.

The cumulative plantar tissue stress was estimated in subjects with diabetic peripheral neuropathy with a history of recurrent ulcers. Surprisingly, it was shown that these patients had lower daily cumulative plantar tissue stress as compared to neuropathic subjects who had never developed a foot ulcer. ${ }^{27}$ Authors have suggested that after an initial episode of skin breakdown, plantar tissue may become more susceptible to future lesions as a result of disuse atrophy following treatment of the first episode, so that plantar tissues re-ulcerate even under low stress. ${ }^{27}$ Thus, the ulcerated subjects in this study may be predisposed to re-ulceration due to their poor biomechanical condition in comparison to the non-ulcerated group.

More alterations in plantar pressure variables were expected than our findings showed. A possible explanation for the lower observed alteration may be that the diabetic patients evaluated may have learned conservational motor strategies for gait (e.g., changing from ankle to hip use to achieve shorter applications of force in the ankle complex, ${ }^{2}$ minimizing plantar loads. Another possible strategy to minimize plantar loads that can be adopted by neuropathic subjects is the redistribution of loads from the more insensate areas to the more sensate, as already described in the litarature. ${ }^{28}$

Notably, the pressure-time integral was the variable that was the most different among groups (Table 2). The pressure-time integral was able to distinguish the neuropathic groups from the control group in all areas studied and to distinguish the neuropathic groups from each other in two out of five areas. This variable may be related to neuropathy progression deficits, and greater changes in this variable may be expected in accordance with the progression of neuropathy. Others ${ }^{6,43-45}$ have reported this variable as a better parameter to evaluate the concept of load than peak pressure, which for the last two decades has been the only biomechanical variable associated with ulcer formation. The pressure-time integral provides an indication of plantar loading behavior over time, and the present study suggests a different mechanism for load absorption and distribution in the neuropathic subjects resulting in a non-homogeneous pattern for all plantar areas, as has been observed in the literature. ${ }^{32}$ Furthermore, the presence of elevated plantar pressure shown by the pressure-time integral can also be associated with other orthopedic and kinesiological factors, such as foot and ankle joint deformities and mobility, which were not the focus of the present study.

Similar coefficients of variation (Table 2) were found by other authors, ${ }^{41}$ where diabetic neuropathy patients did not show higher loading variability when compared to control subjects. However, in agreement with another study that showed higher CVs in neuropathic patients with more advanced neuropathy progression, ${ }^{33}$ this study demonstrated that the group with a previous history of ulceration (DNU) presented higher variability in plantar pressure distribution in comparison to both the neuropathic group without a history of ulceration (DN) and the control group. As the higher variability was more evident at the rearfoot and hallux, we conclude that more stability and motor control are required at the beginning and end of the support phase, and this higher variability may represent an attempt to improve compensations in motor control caused by alterations in gait due to external and internal changes. ${ }^{46}$

The existence of a statistical type II error must be considered, especially because of the sample size of the ulcerated group $(\mathrm{n}=10)$. It is important to highlight that 
during all clinic visits to recruit our neuropathic patients, neuropathic subjects with a history of healed ulceration were heavily outnumbered by patients presenting unhealed wounds and amputation, reflecting the poor quality of diabetes care and follow-up.

Neuropathic subjects with no history of foot ulcers represent patients at an earlier stage of neuropathy than those with a history of foot ulcers. Considering this fact, neuropathy itself first alters plantar pressure distribution, then the ulcers occur, and after healing, the dynamic pattern remains altered and worsens plantar load distribution, predisposing patients to re-ulceration.

\section{CONCLUSIONS}

The present investigation shows that neuropathic groups, both non-ulcerated and ulcerated, presented alterations in plantar pressure distribution patterns, and the ulcerated patients presented higher loads than non-ulcerated. The history of foot ulcers in the clinical history of the diabetic neuropathy subjects influenced plantar pressure distribution, resulting in an increased load under the midfoot and rearfoot and an increase in the variability of plantar pressure during barefoot gait. Progression of diabetic neuropathy could not be explained in this study as influencing plantar pressure distribution, as the groups presented the same clinical signs and symptoms of diabetic neuropathy.

Further longitudinal studies to investigate the presence of other predictive factors for foot ulceration in both neuropathic groups, such as footwear, foot and ankle joint mobility, foot deformities and autonomic and vascular deficits, would contribute to a better understanding of the development of foot ulceration. This knowledge could prevent initial or recurrent foot ulceration in patients with diabetic neuropathy.

\section{ACKNOWLEDGMENTS}

We thank FAPESP for the key funding granted to this project (\# 04/09585-2).

\section{REFERENCES}

1. Cavanagh PR, Simoneau GG, Ulbrecht JS. Ulceration, unsteadiness and uncertainty: the biomechanical consequences of diabetes mellitus. Journal of Biomechanics. 1993;26:S23-40.

2. Mueller JM, Minor SD, Sahrmann SA, Schaaf JA, Strube MJ. Differences in the gait characteristics of patients with diabetes and peripheral neuropathy compared with age-matched controls. Physical Therapy. 1994;74:299-308.

3. Kwon O-Y, Minor SD, Maluf K, Mueller MJ. Comparison of muscle activity during walking in subjects with and without diabetic neuropathy. Gait and Posture. 2003;18:105-13.

4. Sacco ICN, Amadio AC. Influence of the diabetic neuropathy on the behavior of electromyographic and sensorial responses in treadmill gait. Clinical Biomechanics. 2003;18:426-34.

5. Shaw JE, van Shie CHM, Carrington AL, Abbott CA, Boulton AJM. An analysis of dynamic forces transmitted through the foot in diabetic neuropathy. Diabetes Care. 1998;21:1955-9.

6. Maluf KS, Mueller MJ, Strube MJ, Engsberg JR, Johnson JE. Tendon Achilles lengthening for treatment of neuropathic ulcers causes a temporary reduction in forefoot pressure associated with changes in plantar flexor power rather than ankle motion during gait. Journal of Biomechanics. 2004;37:897-906.

7. Masson EA, Hay EM, Stockley I, Veves A, Betts RP, Boulton AJ. Abnormal foot pressures alone may not cause ulceration. Diabetic Medicine. 1989;6:426-8

8. Fernando DJS, Masson EA, Veves A, Boulton AJ. Relationship of limited joint mobility to abnormal foot pressures and diabetic foot ulceration. Diabetes Care. 1991;14:8-11.
9. Payne C, Turner D, Miller K. Determinants of plantar pressure in the diabetic foot. Journal of Diabetes and Its Complications. 2002;16:27783.

10. Bus SA, Maas M, de Lange A, Michels RPJ, Levi M. Elevated plantar pressures in neuropathic diabetic patients with claw/hammer toe deformity. Journal of Biomechanics. 2005;38:1918-25.

11. Stokes IA, Faris IB, Hutton WC. The neuropathic ulcer and loads on the foot in diabetic patients. Acta Orthopaedic Scandinava. 1975;46:83947.

12. Boulton AJ. The importance of abnormal foot pressures in early diabetic neuropathy. Diabetic Medicine. 1987;4:225-8.

13. Rich J, Veves A. Foorfoot and rear foot plantar pressures in diabetic patients: correlation to foot ulceration. Wounds. 2000;12:82-7.

14. Veves A, Murray HJ, Young MJ, Boulton AJM. The risk of foot ulceration in diabetic patients with high foot pressures: a prospective study. Diabetologia. 1992;35:660-3.

15. Frykberg RG, Lavery LA, Pham H, Harvey C, Harkless L, Veves A. Role of neuropathy and high foot pressures in diabetic foot ulceration. Diabetes Care. 1998;21:1714-9.

16. Reiber GE, Smith DG, Wallace C, Sullivan K, Hayes S, Vath C, et al. Effect of therapeutic footwear on foot reulceration in patients with diabetes: a randomized controlled trial. JAMA. 2002;287:2552-8.

17. Feldman EL, Stevens MJ, Thomas PK, Brown MB, Canal N, Greene DA. A practical two-step quantitative clinical and electrophysiological assessment for the diagnosis and staging of diabetic neuropathy. Diabetes Care. 1994;17:1281-9. 
18. Cavanagh PR, Sims JDS, Sanders LJ. Body mass is a poor predictor of peak plantar pressure in diabetic men. Diabetes Care. 1991;14:750-5.

19. Perry JE, Hall JO, Davis BL. Simultaneous measurement of plantar pressure and shear forces in diabetic individuals. Gait and Posture. 2002;15:101-7.

20. Armstrong DG, Lavery LA. Elevated peak plantar pressure in patients who have Charcot arthropathy. The Journal of Bone and Joint Surgery. 1998;80:365-9.

21. Hsiao H, Guan J, Weatherly M. Accuracy and precision of two in-shoe pressure measurement systems. Ergonomics. 2002;45:537-55.

22. Burnifield JM, Few CD, Mohamed OS, Perry J. The influence of walking speed and footwear on plantar pressures in older adults. Clinical Biomechanics. 2004;19:78-84.

23. Cavanagh PR, Ulbrecht JS. Biomechanical aspects of foot problems in diabetes. In: Boulton AJM, Connor H, Cavanagh PR. The foot in diabetes. Ed. Chichester, United Kingdom; 1994. p. 25-35.

24. Lucarelli PR, Lima MdeO, Lucarelli JG, Lima FP. Changes in joint kinematics in children with cerebral palsy while walking with and without a floor reaction ankle-foot orthosis. Clinics. 2007;62:63-8

25. Lucarelli PR, D'Andrea Greve JM Knee joint dysfunctions that influence gait in cerebrovascular injury. Clinics. 2008;63:443-50

26. Bacarin TA, Akashi PMH, Sacco ICN. Duration of disease, neuropathic symptoms, and plantar sensitivity in patients with diabetes with and without previous plantar ulceration. Wounds. 2008;20:37-45.

27. Maluf KS, Mueller MJ. Comparison of physical activity and cumulative plantar tissue stress among subjects with and without diabetes mellitus and a history of recurrent plantar ulcers. Clinical Biomechanics. 2003;18:567-75.

28. Nurse MA, Nigg BM. The effect of changes in foot sensation on plantar pressure and muscle activity. Clinical Biomechanics. 2001;16:71927.

29. Eils E, Noltea S, Tewesa M, Thorwestenb L, Volkerb K, Rosenbaum D. Modified pressure distribution patterns in walking following reduction of plantar sensation. Journal of Biomechanics. 2002;35:1307-13.

30. Taylor A, Menz HB, Keenan A-M. Effects of experimentally induced plantar insensitivity on forces and pressures under the foot during normal walking. Gait and Posture. 2004;20:232-7.

31. Cavanagh PR, Rodgers MM. The Arch Index: a useful measure from footprints. Journal of Biomechanics. 1987;20:547-51.

32. Giocomozzi C, Caselli A, Macellari V, Giurato L, Lardieri L, Uccioli L. Walking strategies in diabetic patients with peripheral neuropathy. Diabetes Care. 2002;25:1451-7.
33. Sacco ICN, Amadio AC. A study of biomechanical parameters in gait analysis and sensitive cronaxie of diabetic neuropathic patients. Clinical Biomechanics. 2000;15:196-202.

34. Bus SA, Maas M, Lange A, Michels RPJ, Levi M. Elevated plantar pressures in neuropathic diabetic patients with claw/hammer toe deformity. Journal of Biomechanics. 2005;38:1918-25.

35. Fernando DJS, Masson EA, Veves A, Boulton AJM. Relationship of limited joint mobility to abnormal foot pressures and diabetic foot ulceration. Diabetes Care. 1991;14:8-11.

36. Salsich GB, Mueller MJ, Sahrmann SA. Passive ankle stiffness in subjects with diabetes and peripheral neuropathy versus an age matched comparison group. Phys Ther. 2000; 80:352-62.

37. Pham H, Armstrong DG, Harvey C, Harkless LB, Giurini JM, Veves A. Screening techniques to identify people at high risk for diabetic foot ulceration: a prospective multicenter trial. Diabetes Care. 2000;23:60611.

38. Gefen A. Plantar soft tissue loanding under the medial metatarsals in the standing diabetic foot. Medical Engineering \& Physics. 2003;25:4919.

39. Turner DE, Helliwell PS, Burton AK, Woodburn J. The relationship between passive range of motion and range of motion during gait and plantar pressure measurements. Diabet Med. 2007;24:1240-6.

40. Sinacore DR, Bohnert KL, Hastings M, Johnson JE. Mid foot kinetics characterize structural polymorphism in diabetic foot disease. Clinical Biomechanics. 2008;23:653-61.

41. Cavanagh PR, Preey JE, Ulbrecht JS, Derr JA, Pammer SE. Neuropahatic diabetic patients do not have reduced variability of plantar loading gait. Gait and Posture. 1998; 7:191-9.

42. Duckworth T, Boulton AJM, Betts RP, Franks CI, Ward JD. Plantar pressure measurements and the prevention of ulceration in the diabetic foot. Journal of Bone and Joint Surgery. 1985;67:79-85.

43. Shaw JE, Boulton AJM. Pressure time integrals may be more important than peak pressures in diabetic foot ulceration. Diabet. Med. 1996;13:S22.

44. Sauseng S, Kastenbauer T, Sokol G, Irsigler K. Estimation of risk for plantar foot ulceration in diabetic patients with neuropathy. Diabetes Nutrition and Metabolic. 1999; 12:189-93.

45. Cavanagh PR, Ulbrecht JS, Caputo G. New developments in the biomechanics of the diabetic foot. Diabetes Metab. Res. Rev. 2000;16:S6-10.

46. Kimmeskamp S, Hennig EM. Heel to toe motion characteristics in Parkinson patients during free walking. Clinical Biomechanics. 2001;16:806-12. 